\title{
The IDIPOS project: is a multidisciplinary data infrastructure for weather and space weather feasible?
}

\author{
Vincenzo Romano ${ }^{1,{ }^{\star}}$, Luca Spogli ${ }^{1}$, Alberto Salvati ${ }^{2}$, Claudio Rafanelli ${ }^{3}$, Lili Cafarella ${ }^{1}$ \\ ${ }^{1}$ Istituto Nazionale di Geofisica e Vulcanologia, Sezione Roma 2, Rome, Italy \\ ${ }^{2}$ Consiglio Nazionale delle Ricerche, Ufficio Sistemi Informativi e Documentali, Rome, Italy \\ ${ }^{3}$ Consiglio Nazionale delle Ricerche, Istituto di Acustica e Sensoristica 'Orso Mario Corbino', Rome, Italy
}

Article history

Received November 13, 2012; accepted March 27, 2013.

Subject classification:

Database, IT infrastructure, Polar observation, Space weather, Interoperability.

\begin{abstract}
The Italian National Antarctic Programme (PNRA) has long supported scientific and technological activities addressed to the implementation, upgrade and maintenance of infrastructure and instruments supporting geosciences and physical sciences in the polar region. This report describes the first results of the Italian Database Infrastructure for Polar Observation Sciences (IDIPOS) project, which was recently approved by the PNRA. The project aims to study the feasibility of the hardware and software infrastructure for the construction of relational databases of acquisitions from past and current experiments in the polar areas. The study is based on the fundamental characteristics of the infrastructure: its implementation in Italy, with locations across the country, and its integration into the existing and future telematics infrastructure that will be available at the Italian bases. The project proposes a modern and hightech infrastructure dedicated to data treatment, accessibility and archiving, in line with international standards. This infrastructure will allow modern, rapid and reliable treatment of the acquired data. The IDIPOS infrastructure is crucial during the planning phase of scientific and monitoring activities of the PNRA, especially of those included in the Scientific Committe for Antarctic Research (SCAR) projects, the International Polar Year, and the framework of international cooperation. In the first phase of the project, the observations are related to research projects in different PNRA sectors. The infrastructure will be potentially extendable to other observational activities. In this report, the infrastructure is introduced, while highlighting its usefulness for weather and space-weather forecasting for the scientific community and in the framework of Global Navigation Satellite System (GNSS) Research and Application for Polar Environment (GRAPE) in particular.
\end{abstract}

\section{Introduction}

Data provided by permanent observatories and by automatic stations in the polar regions are of crucial importance to understand the spatial and temporal evolution of the physical and chemical phenomena of the Earth. Knowledge of the global change mech- anism, for instance, is based on long data series of chemical, physical and environmental parameters that have been collected by several observatories and monitoring stations. These long series represent a precious collection of information, and the Italian Antarctic Programme (PNRA) has long supported and funded scientific and technological activities addressed to the implementation, up-grading and maintenance of infrastructure and instruments dedicated to the geosciences and physical sciences in the polar regions. However, the observational activities still lack appropriate services regarding data handling. The establishment of modern, high-tech infrastructure dedicated to data treatment, accessibility and archiving in agreement with international standards is necessary. A plan of such infrastructure that allows modern, rapid and reliable treatment of the acquired data is crucial during the planning phase of the scientific and monitoring activities of the PNRA, especially of those included in SCAR projects and the International Polar Year. Through the synergy that has matured within the Global Navigation Satellite System (GNSS) Research and Application for Polar Environment (GRAPE) Expert group (www.grape.scar.org), which is funded by the Scientific Committe for Antarctic Research (SCAR), a fruitful and close collaboration was born among different groups involved in weather and space-weather forecasting. Several projects inside the Italian Database Infrastructure for Polar Observation Sciences (IDIPOS) deal with weather and spaceweather research, with these IDIPOS concepts being very useful for this community, due to the interoperations among tools and data sharing. The full list of the research projects involved in the IDIPOS project are summarized in Table 1. 


\begin{tabular}{|c|c|c|c|}
\hline PNRA sector & Original title & English title (not official) & $\begin{array}{l}\text { Principal } \\
\text { investigator }\end{array}$ \\
\hline \multirow{6}{*}{$\begin{array}{l}\text { Geodesy and } \\
\text { Observatories }\end{array}$} & $\begin{array}{l}\text { Osservatori sismologici permanenti } \\
\text { in Antartide }\end{array}$ & $\begin{array}{l}\text { Permanent seismological observatories } \\
\text { in Antarctica }\end{array}$ & Morelli \\
\hline & Osservatorio Meteo-Climatologico Antartico & $\begin{array}{l}\text { Antarctic Observatory for Weather and Cli- } \\
\text { mate }\end{array}$ & $\begin{array}{l}\text { Pellegrini / } \\
\text { Grigioni }\end{array}$ \\
\hline & $\begin{array}{l}\text { Osservazioni in alta atmosfera } \\
\text { e climatologia spaziale }\end{array}$ & $\begin{array}{l}\text { Observations in the upper atmosphere } \\
\text { and Space Weather }\end{array}$ & De Franceschi \\
\hline & $\begin{array}{l}\text { Misure accurate dei flussi di radiazione solare } \\
\text { ed infrarossa alla superficie presso la Base Mario } \\
\text { Zucchelli e a Dome C (stazione BSRN) }\end{array}$ & $\begin{array}{l}\text { Accurate measurements of the solar } \\
\text { and infrared radiation fluxes at the surface } \\
\text { at the Mario Zucchelli Station and Dome C }\end{array}$ & Lanconelli \\
\hline & $\begin{array}{c}\text { Osservatori Sismici tra Concordia e Vostok } \\
\text { per lo studio della struttura litosferica } \\
\text { e profonda della Terra }\end{array}$ & $\begin{array}{l}\text { Seismic Observatories between Concordia } \\
\text { and Vostok for the study of the lithospheric } \\
\text { and deep Earth structure }\end{array}$ & Danesi \\
\hline & $\begin{array}{l}\text { Osservazioni di Geomagnetismo } \\
\text { ed Elettromagnetismo in Antartide }\end{array}$ & $\begin{array}{l}\text { Observations of Geomagnetism } \\
\text { and Electromagnetism in Antarctica }\end{array}$ & Cafarella \\
\hline \multirow{2}{*}{ Geophysics } & Archivio web based dei dati antartici & Web based archive of Antarctic data & Wardell \\
\hline & Antarctic Seismic Data Library System (SDLS) & $\begin{array}{l}\text { Antarctic Seismic Data Library System } \\
\text { (SDLS) }\end{array}$ & Diviacco \\
\hline Glaciology & Permafrost e Cambiamento Climatico & Permafrost and Climate Change & Guglielmin \\
\hline \multirow{2}{*}{$\begin{array}{l}\text { Physics and } \\
\text { Chemistry of } \\
\text { the Atmosphere }\end{array}$} & $\begin{array}{l}\text { Effetti radiativi diretti di aerosol e nubi sottili } \\
\text { alle alte latitudini: una prospettiva bipolare } \\
\text { (DECA-POL). }\end{array}$ & $\begin{array}{l}\text { Direct radiative effects of aerosols and thin } \\
\text { clouds at high latitudes: a bipolar perspective } \\
\text { (DECA-POL). }\end{array}$ & Vitale \\
\hline & $\begin{array}{c}\text { I gas atmosferici minori e la radiazione solare UV } \\
\text { quali fattori d'impatto climatico: } \\
\text { modelli e campionamento }\end{array}$ & $\begin{array}{l}\text { The minor atmospheric gases and solar } \\
\text { radiation UV as climate impact factors: } \\
\text { models and sampling }\end{array}$ & Rafanelli \\
\hline \multirow{4}{*}{$\begin{array}{l}\text { Solar-Terrestrial } \\
\text { relationship and } \\
\text { Astrophysics }\end{array}$} & $\begin{array}{l}\text { Studio bipolare di fenomeni aurorali con i radar } \\
\text { SuperDARN e con osservazioni ottiche }\end{array}$ & $\begin{array}{l}\text { Bipolar study of auroral phenomena with Su- } \\
\text { perDARN radar and optical observations }\end{array}$ & Amata \\
\hline & Stazione Groenlandese per Osservazioni aurorali & Greenland station for auroral observations & Massetti \\
\hline & $\begin{array}{c}\text { Rete magnetometrica italiana } \\
\text { nell'ambito di AIMNet (Antarctic International } \\
\text { Magnetometer Network) }\end{array}$ & $\begin{array}{c}\text { Italian magnetometer network } \\
\text { within AIMNet (Antarctic International } \\
\text { Magnetometer Network) }\end{array}$ & Lepidi \\
\hline & $\begin{array}{l}\text { Raggi cosmici in aree polari } \\
\text { e fenomeni terrestri associati. }\end{array}$ & $\begin{array}{l}\text { Cosmic rays in the polar areas } \\
\text { and associated terrestrial phenomena. }\end{array}$ & Storini \\
\hline Technology & $\begin{array}{l}\text { Radiometro UV a filtri per la misura } \\
\text { dell'irradianza solare diretta e diffusa e di quella } \\
\text { biologicamente efficace presso le Stazioni Mario } \\
\text { Zucchelli (SMZ) e Dome Concordia }\end{array}$ & $\begin{array}{l}\text { UV filter radiometer for measuring direct } \\
\text { and diffuse solar radiance and biologically } \\
\text { effective at Mario Zucchelli Station (SMZ) } \\
\text { and Dome Concordia }\end{array}$ & Scaglione \\
\hline \multirow{2}{*}{ Arctic } & $\begin{array}{l}\text { ITACA2: network per le osservazioni aurorali } \\
\text { congiunte ad alta latitudine. }\end{array}$ & $\begin{array}{l}\text { ITACA2: a network for the joint high-latitude } \\
\text { auroral observations. }\end{array}$ & Massetti \\
\hline & $\begin{array}{l}\text { ISACCO (Ionospheric Scintillation Arctic } \\
\text { Coordinated Campaigns Observations) }\end{array}$ & $\begin{array}{l}\text { ISACCO (Ionospheric Scintillation Arctic } \\
\text { Coordinated Campaigns Observations) }\end{array}$ & De Franceschi \\
\hline
\end{tabular}

Table 1. Research projects involved in IDIPOS. 


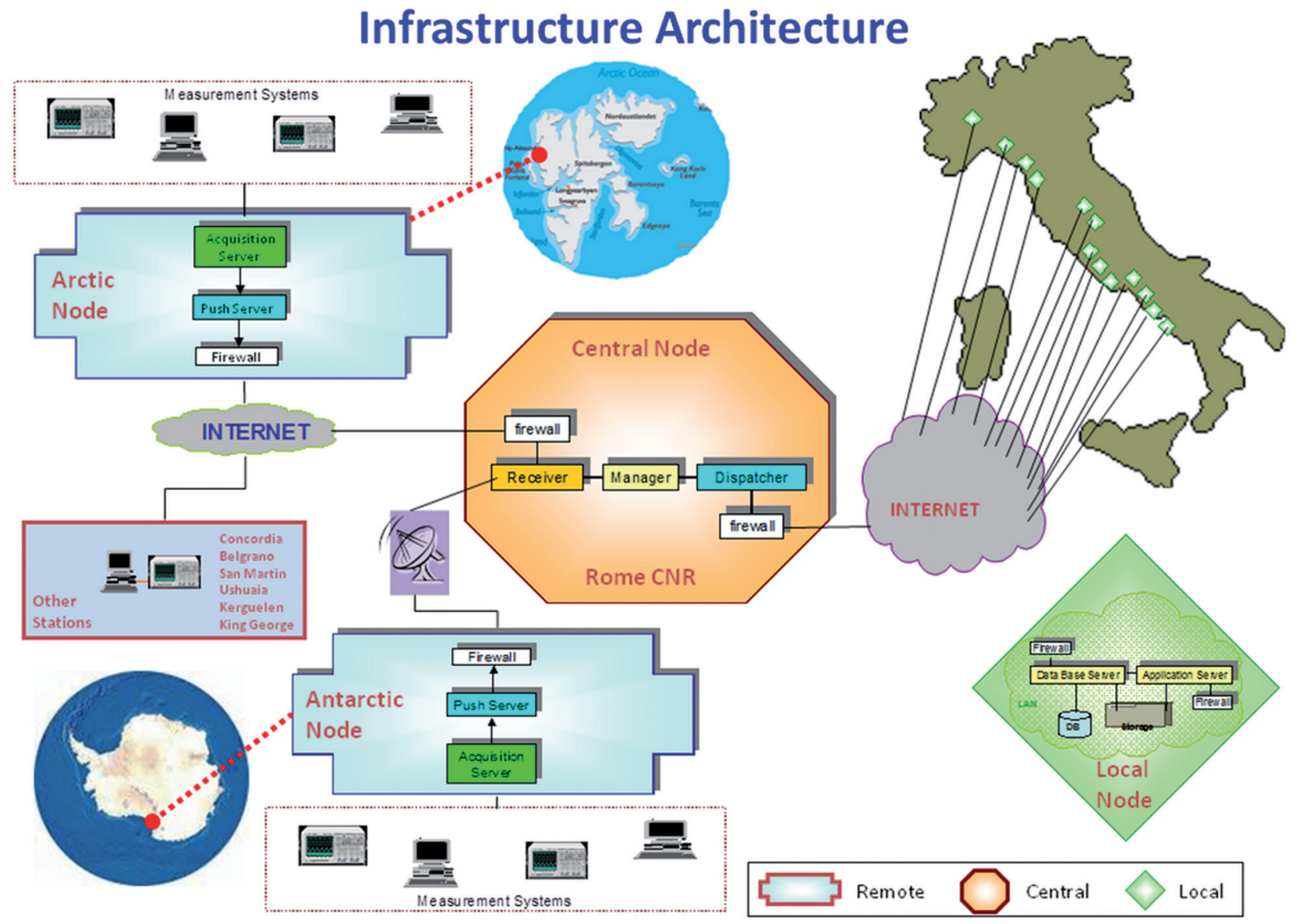

Figure 1. Infrastructure architecture of the IDIPOS system.

\section{System concepts}

The aim of IDIPOS is to perform a feasibility study for a hardware and software structure that can guarantee transmission, processing and storing of raw and processed data from the polar observatories. The feasibility study was developed to guarantee a distributed database architecture throughout the whole Italian territory. Every research group has a dedicated system to handle safety, processing and data accessibility.

The whole infrastructure architecture of the IDIPOS is illustrated in Figure 1, and it is based on a network in which each node is a complex hardware and software system that can automatically process information. The Antarctic Node at the 'Mario Zucchelli' Italian Antarctic Station (MZS), and the Arctic Node at the 'Dirigibile Italia' base of the Italian National Research Council (CNR) located at the Svalbard Islands, consist of two elements: the 'acquisition server', to collect information coming from different measurement stations; and the 'push server', to handle data organization and transmission to Italy. Every project takes care of the sending of data to the correct polar node. The architecture of the Central Node in Italy is based on three systems: the 'receiver', the 'manager', and the 'dispatcher'. The receiver handles the data flow coming from the polar nodes, according to different formats, protocols, channels and bandwidth. The manager optimizes the information to be sent to the local nodes, to provide information about data flow and remote instruments status. The dispatcher sends the data to the local nodes in Italy.

The Local Node, as illustrated in Figure 2, is based on a 'database server' and the 'storage' for data handling and storing, and the 'application server' that manages the application logic. Among the different tasks of the local nodes, there are: data receiving, data conversion, database up-dating, handling and processing, output production, website hosting, user interface, and data back-up.

Communication between the central and local nodes will use the national net; communication with polar areas will rely on the present or future available systems at the polar bases.

\subsection{Objectives and advantages}

The feasibility study is multi-disciplinary and multi-instrumental, and it concerns the original infrastructure through which different research sectors can interoperate. The infrastructure has the possibility to be integrated with other data types that are not present in this feasibility study phase. The complete automation and data treatment provides each research 


\section{Infrastructure Architecture}

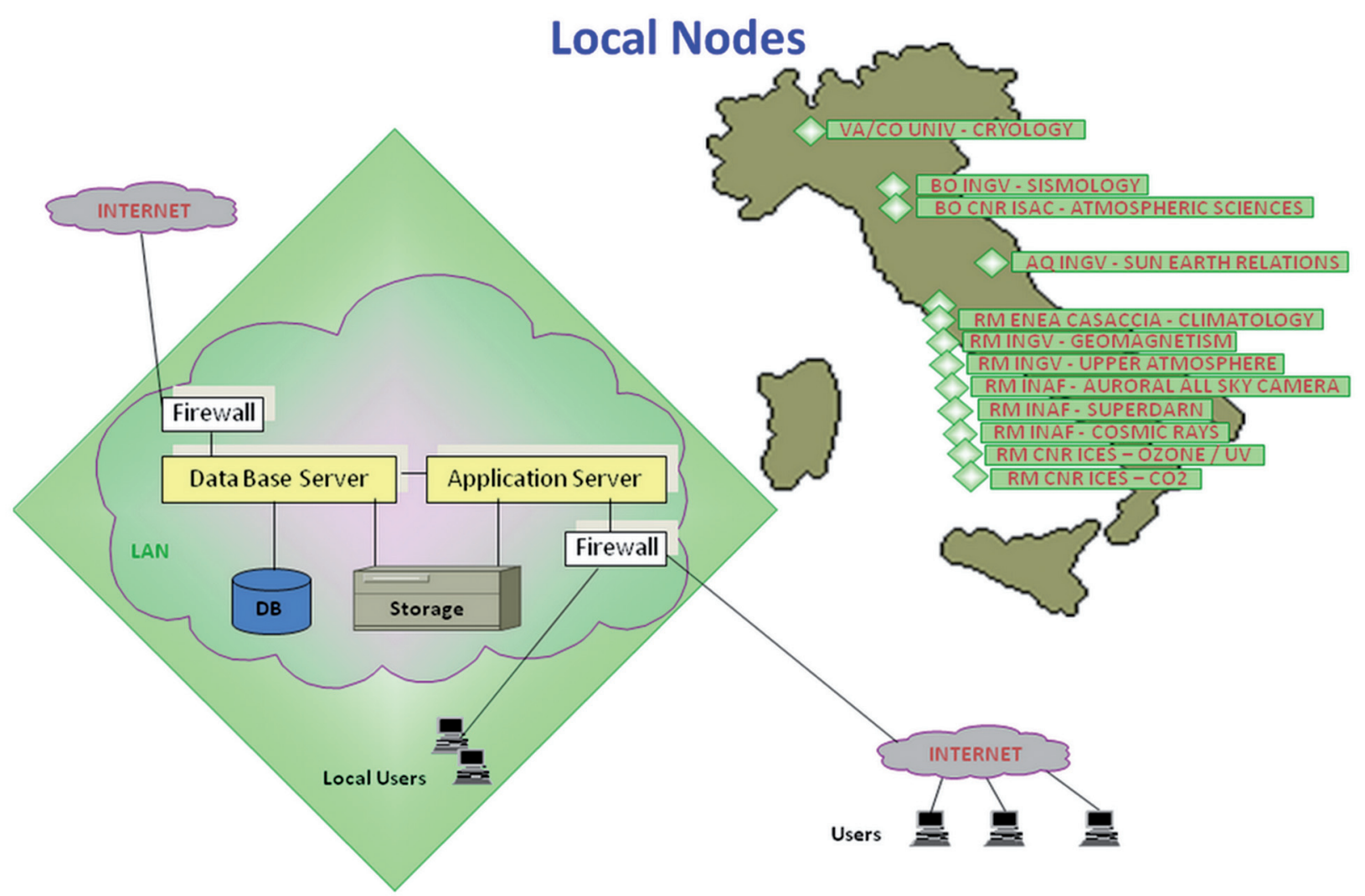

Figure 2. Infrastructure architecture of the IDIPOS Local Nodes.

project with always-updated archives and ensures realtime checking of the remote instrument status, through a dedicated website. These features are necessary to satisfy the rapid access to the data and their derived products. The infrastructure is designed to fulfill the following main objectives:

- A feasibility study of the basic infrastructure of the hardware and software. The infrastructure allows the creation of relational databases that contain digital data that was acquired during present and past measurement campaigns in the polar areas.

- A feasibility study of the tools for the data treatment. Dynamic tools for data treatment are produced. These operate in a protected environment, and allow automatic up-dating, processing and archiving of any kind of research project data.

- A feasibility study dedicated to the integration between IDIPOS and the current Italian National Antarctic Data Centre (NADC) (for more details of the Italian NADC infrastructure for metadata accessibility, see Benedetti et al. [2006] and Damiani et al. [2008]). The study guarantees the interoperability between IDIPOS and the Italian NADC.

- An analysis of the added value to research projects e.g., in terms of improvements to research activities.

- Structuring of historical and real-time data.

- Making any experimental and research activity more visible, due to the data accessibility.

- Interoperability with national and international projects, for exchange and sharing of information.

- Availability of high-quality and high-tech standard tools.

- Implementation of a demonstrator showing some of the functionality, for a limited set of data.

\subsection{Technical requirements}

The main infrastructure requirements that are taken into account in this feasibility study are:

1. Simplicity of use. Users are provided with intuitive and simple tools.

2. Interactivity. The quality concerning response times and messages is guaranteed. To maintain a small delay introduced by the network, the quantity of information is minimized.

3. Scalability, flexibility and expandability. The system can respond to the increasing number of users, and the increasing diversification of the data.

4. Standard and interoperability. Particular attention is used to follow the main standards for high interoperability with existing national and international projects.

5. Compatibility. The system works in the same way on different platforms (Unix, Windows, Macintosh).

6. Multi user management. Access is allowed to dif- 
ferent users, simultaneously.

7. Remote management. Administrator remote access is possible for database handling.

8. Storing information capability and formats. Data resulting from measurements are stored in different formats, including audio, video, and the most popular formats (PostScript, PDF, Word, ZIP).

9. Output capability. Database structured data is retrieved and presented in different popular formats (text, HTML, XML).

10. Web updating and publishing. The system updates information to avoid differences between the database and the website.

11. Users profile and accessibility. Reserved information is protected. Users have the correct privileges to access.

12. Access, profiles, user management (data policy). Every local system has an administrator who manages the different privileges and profiles.

13. Automatic control and production of information statistics. Every local system is automatically controlled to monitor data flow and processing, to produce informative statistics.

14. Infrastructure maintenance. Maintenance of the infrastructure is guaranteed during and after the implementation phase.

\section{International context}

Following the strategic recommendation of SCAR [Finney 2009], IDIPOS is planned to support the building of an Antarctic Data Management System (ADMS) that is capable of supporting Antarctic science and SCAR activities within the Antarctic Treaty System. The ADMS should be viewed as a science enabler and a service that supports practical applications of various kinds. As reported in the recommendations, IDIPOS will maintain the following international strategic standards:

a. Articulated governance arrangements and strong leadership, suitable for driving the development of a distributed, but loosely federated, shared infrastructure.

b. Fostering of a culture willing to share and collaborate in data-management-related activities.

c. Leveraging of existing SCAR and non-SCAR systems, capabilities and resources, and supplementation of these where there are obvious deficiencies (the primary purpose of this leveraging would be to create a network of designated permanent data archives that are capable of the long-term management and publication of all types of SCAR-related data).

d. Agreement on and implementation of standards that support the interoperation of technology platforms and data-transport protocols. In particular, the development or adoption of standards to describe and encode data objects, equipment, processing techniques and instruments that ultimately function to permit data integration and aggregation.

e. Education, outreach and guidance on all facets of the system operation, protocols and functions.

This project directly supports some of the research project activities in the framework of international cooperation, such as:

- Standing Committee on Antarctic Data Management (SC-ADM; www.scadm.scar.org);

- GRAPE SCAR Expert Group (www.grape.scar. org);

- Interhemispheric Conjugacy Effects in Solar-Terrestrial and Aeronomy Research (ICESTAR; http://scar -icestar.org/);

- Antarctic and Sub-Antarctic Permafrost, Soils and Periglacial Environments Group (ANTPAS; http:/ / classic.ipy.org/development/eoi/proposal-details.php? $\mathrm{id}=50$ );

- Svalbard Integrated Arctic Earth Observing System (SIOS; http:/ / www.sios-svalbard.org).

\section{Case study}

In this section, we report on a case study in which the multidisciplinary potentiality of the IDIPOS system is applied to the case of a geomagnetic event that was observed in late April 2011 in Antarctica. In particular, observations recorded by the geomagnetic observatory at Mario Zucchelli Station ( $\left.74^{\circ} 41^{\prime} 42^{\prime \prime S}, 164^{\circ} 07^{\prime} 23^{\prime \prime} E\right)$, in the framework of the project Observations of Geomagnetism and Electromagnetism in Antarctica, are compared with those acquired through co-located Global Positioning System Ionospheric Scintillation and Total Electron Content (TEC) Monitor (GISTM) receivers, in the framework of the project Observations in the Upper Atmosphere and Space Weather.

The geomagnetic observatory has been operating since the austral summer of 1986-87 [Meloni et al. 1997]. Variations in the Earth magnetic field are measured through three-axis fluxgate magnetometers. The field variations are measured along three directions that are oriented with reference to the local magnetic meridian, respectively: the horizontal magnetic field intensity H component (south-north); the orthogonal-component $\mathrm{D}$ in the horizontal plane (west-east, whereby $\mathrm{D}$ is an intensive element, expressed consequently in $\mathrm{nT}$, and not the angular element declination); and the vertical intensity Z-component (consequently with positive increase inwards).

The GISTM receivers are special receivers that can measure the ionospheric vertical (v)TEC and the phase and amplitude scintillation indices $\left(\sigma_{\Phi}\right.$ and $\left.S_{4}\right)$, 


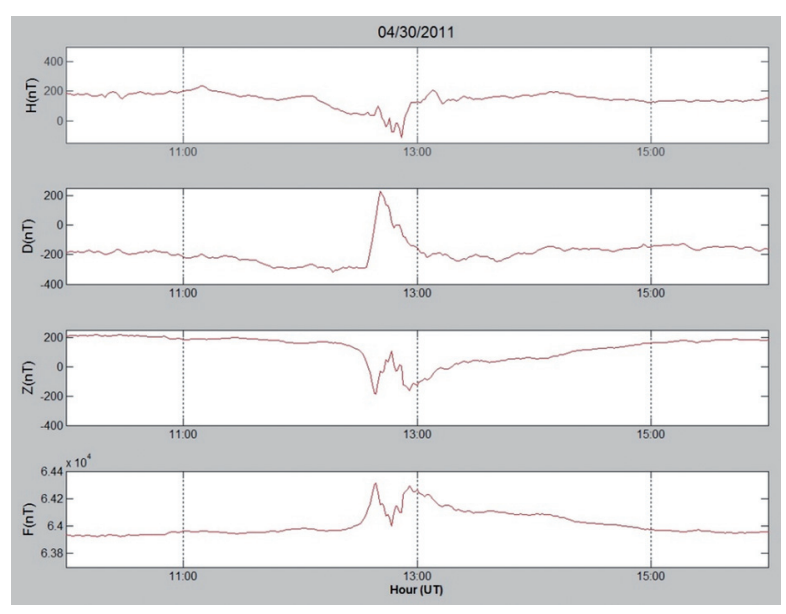

Figure 3. Time series of the horizontal $(\mathrm{H})$, declination $(\mathrm{D})$ and $\mathrm{Z}$ (into Earth) components, and total field intensity (F), measured at the Mario Zucchelli Station.

by sampling the GPS signal at $50 \mathrm{~Hz}$ [Van Dierendonck et al. 1993]. This GISTM receiver is also part of the network of the GRAPE SCAR Expert Group. The data of each instrument are structured into separate databases and are accessible to users through the web.

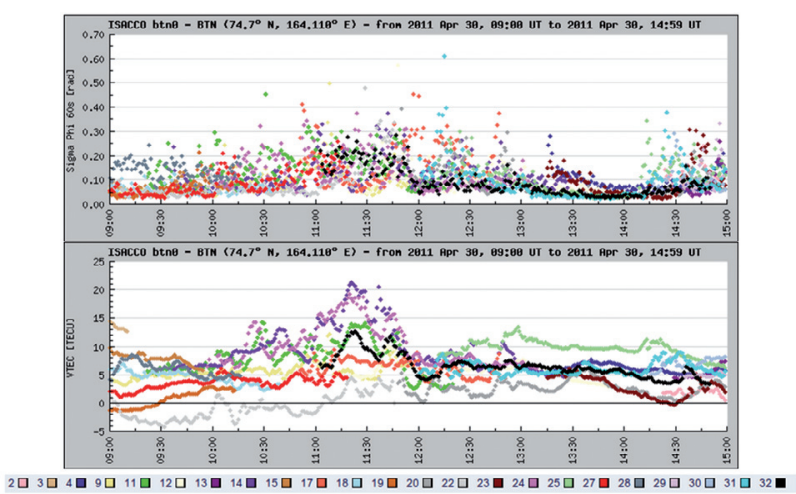

Figure 4. Time evolution of the phase scintillation index $\sigma_{\Phi}$ (top) and vTEC (bottom) in the time range of 09:00 to 15:00 UT of the date considered, which are available from the eSWua database (www.eswua.ingv.it). Each color corresponds to a different satellite, according to the code at the base of the figure.

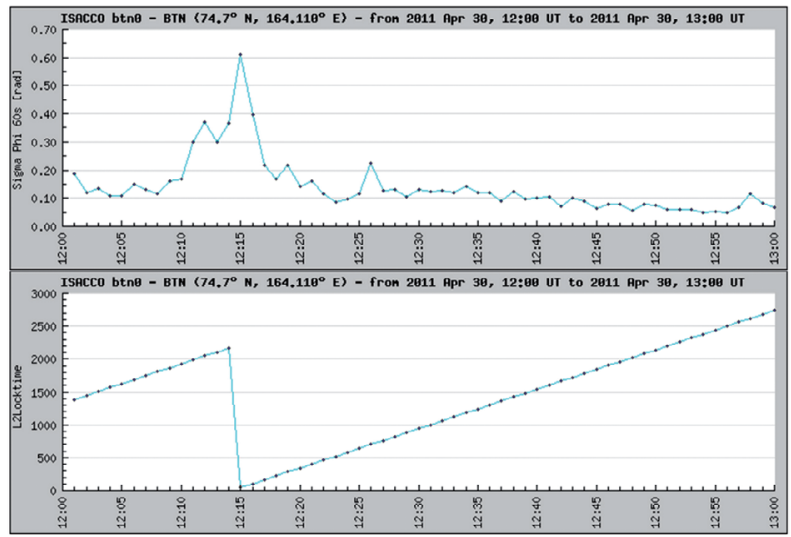

Figure 5. Time evolution of the phase scintillation index $\sigma_{\Phi}$ (top) and locktime of the L2 signal (bottom) in the time range 12:00 to 13"00 UT of GPS satellite 31.
Geomagnetic data and plots are available at http:/ / geo mag.rm.ingv.it. The GISTM data and plots are available at the electronic space weather upper atmosphere (eSWua) database (www.eswua.ingv.it; Romano et al. [2008]), in which all of the data acquired by the upper atmosphere instruments of the Istituto Nazionale di Geofisica e Vulcanologia (INGV) are structured and accessible. As a possible application of the interoperability among the two databases envisaged by IDIPOS, we report on the way in which the simultaneous use of both of these datasets allows a deeper understanding of the conditions of the near-Earth magnetic environment, possibly leading to ionospheric scintillation of GNSS signals.

A solar wind stream originated from a coronal hole hit the Earth magnetic field during the early hours of April 30, 2011, and it generated a system of local electric currents that were observed at high latitudes. Figure 3 shows the time evolution of the horizontal $(\mathrm{H})$, declination $(\mathrm{D})$ and $Z$ components of the geomagnetic field, together with the total field intensity $(\mathrm{F})$ measured at Mario Zucchelli Station between 09:00 and 15:00 UT. In a few minutes, the values of the geomagnetic components change abruptly, by $250 \mathrm{nT}$ in the case of $\mathrm{H}$, and about $400 \mathrm{nT}$ in the case of the other two components. Also, the intensity of the geomagnetic field went from about $64000 \mathrm{nT}$ to $64400 \mathrm{nT}$. Figure 4 shows the time series of the phase scintillation index $\sigma_{\Phi}$ (top) and the vTEC (bottom) in the time range of 09:00 to 15:00 UT, as available from eSWua for that day. In Figure 4, each color corresponds to a different satellite, according to the code at the base of the Figure. By comparing Figure 3 and Figure 4, it is possible to highlight an overall steep enhancement of the vTEC (up to a few tenths of a TECU) of all of the satellites in correspondence with the early phases of the geomagnetic event. Such steep gradients lead to larger values of the phase scintillation index, which can possibly corrupt the availability of the GPS signals (see, e.g. De Franceschi et al. [2008]). In particular, Figure 5 shows the time evolution of the phase scintillation index $\sigma_{\Phi}$ (top) and locktime of the L2 signal (bottom) in the time range 12:00 to 13:00 UT of the GPS satellite 23. In this case, it is evident how the ionospheric scintillation that are driven by ionospheric electrical currents can induce the loss of the lock of the signals, which will jeopardizing the reliability of the GNSS service. Figure 6 shows a polar plot of the phase scintillation index $\sigma_{\Phi}$ in the time range of 09:00 to 15:00 UT, to identify the geographic sector in which the geomagnetic disturbance measured by the Mario Zucchelli Station magnetometers drives the ionospheric scintillation phenomenon. 


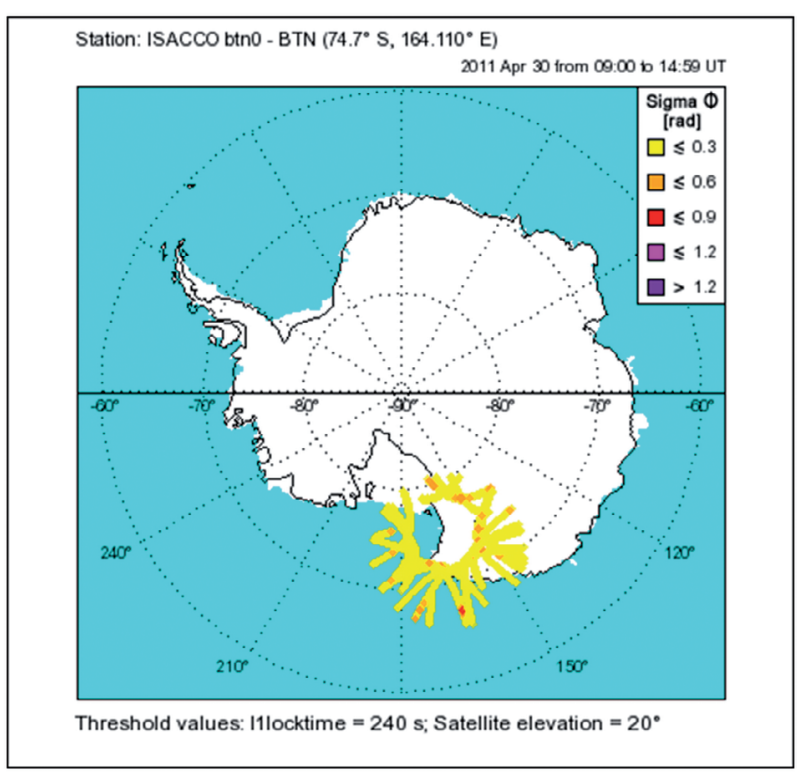

Figure 6. Polar plot of the phase scintillation index $\sigma_{\Phi}$ in the time range 09:00 to 15:00 UT of the data considered, which are available from the eSWua database. Color codes indicate different ranges of $\sigma_{\Phi}$ values.

\section{Summary}

The IDIPOS project aims to realize a feasibility study of a sophisticated hardware and software infrastructure that will allow the creation of relational databases of digital acquisitions from past and current experimental measurements that have been performed in the polar areas of both hemispheres through Italian research projects. The idea of IDIPOS is to foster and share data and tools of the large and multidisciplinary community that is involved in the Earth sciences. IDIPOS will integrate tools and data related to Italian observatories and instruments in the Arctic and Antarctica. The study is conducted according to the most recent recommendations and innovation roadmap endorsed in the ADMS vision, and is also designed to expand the usability of IDIPOS to the international context. In particular, the application of the IDIPOS potentiality is envisaged in the framework of the weather and spaceweather forecasting community, and especially in the activities of the GRAPE SCAR Expert Group. To present a possible application, we report on a case study in which geomagnetic and ionospheric scintillation data were acquired through different instruments and are available on different web services; here, they are used to characterize a geomagnetic event that occurred over Antarctica in late April 2011. The case considered shows how such simultaneous use of data, which is feasible according to the IDIPOS vision, allows the rapid representation of the conditions of the near-Earth magnetic environment that might lead to ionospheric scintillation of the GNSS signals.

A multidisciplinary and interoperable data infrastructure for weather and space weather is feasible, and from this point of view, IDIPOS is a step towards the next level of data sharing and integration maturity for the polar sciences, first at the national level, and then at the international level, which is data management culture and governance.

Acknowledgements. The authors thank the Programma Nazionale di Ricerche in Antartide (PNRA - Italian National Program for Antarctic Research) and the Scientific Committee for Antarctic Research (SCAR); Dott. Domenico Di Mauro, for his contribution to the development of the database "geomagnetism" in the framework of the IDIPOS activities.

\section{References}

Benedetti, E., A. Damiani, C. Rafanelli, M. Castorina, O. Salvetti, M. Storini, L. Testa, V. Vitale and M. Martinelli (2006). Information system for Italian Polar research, In: Proceedings of $21^{\text {st }}$ Polar Libraries Colloquy (Rome, May 8-12), 211-224.

Damiani, A., E. Benedetti, M. Storini and C. Rafanelli (2008). Italian polar data center for capacity building associated with the IHY, Adv. Space Res., 41, 223-226.

De Franceschi, G., L. Alfonsi, V. Romano, M.H.O. Aquino, A. Dodson, C.N. Mitchell and A.W. Wernik (2008). Dynamics of high latitude patches and associated small scale irregularities, J. Atmos. Sol.-Terr. Phys., 70, 879-888; doi:10.1016/j.jastp.2007.05.018.

Finney, K. (2009). Data and Information Management Strategy (DIMS) 2009-2013, SCAR report No 34, September 2009, ISSN 1755-9030; available at http: / / www.scar.org/publications/reports/Report_34.pdf.

Meloni, A., P. Palangio, L. Cafarella, G. Romeo, E. Bozzo and G. Caneva (1997). The geomagnetic observatory at Terra Nova Bay Base, Terra Antarctica, 1, 181-183.

Romano, V., S. Pau, M. Pezzopane, E. Zuccheretti, B. Zolesi, G. De Franceschi and S. Locatelli (2008). The electronic space-weather upper atmosphere (eSWua) project at INGV: advancements and state of the art, Annales Geophysicae, 26, 345-351.

Van Dierendonck, A.J., J. Klobuchar and Q. Hua (1993). Ionospheric scintillation monitoring using commercial single frequency $\mathrm{C} / \mathrm{A}$ code receivers, In: ION GPS-93 Proceedings of the Sixth International Technical Meeting of the Satellite Division of the Institute of Navigation (Salt Lake City, U.S.A., September 22-24), 1333-1342.

\footnotetext{
${ }^{\star}$ Corresponding author: Vincenzo Romano, Istituto Nazionale di Geofisica e Vulcanologia, Sezione Roma 2, Rome, Italy; email: vincenzo.romano@ingv.it.
}

(C) 2013 by the Istituto Nazionale di Geofisica e Vulcanologia. All rights reserved. 